\title{
2D Monolayer of 1T' Phase of Alloyed WSSe from Colloidal Synthesis
}

Ashkan Shahmanesh ${ }^{1}$, Davide Romanin², Corentin Dabard², Sang-Soo Chee ${ }^{2}$, Charlie Gréboval², Christophe Methivier ${ }^{3}$, Mathieu G. Silly ${ }^{4}$, Julien Chaste ${ }^{5}$, Matthieu Bugnet ${ }^{6}$, Debora Pierucci ${ }^{5}$, Abdelkarim Ouerghi ${ }^{5}$, Matteo Calandra ${ }^{2,7,8}$, Emmanuel Lhuillier $^{2^{*}}$, Benoit Mahler $^{1^{*}}$

1 Institut Lumière-Matière, CNRS UMR 5306, Université Lyon 1, Université de Lyon, 69622 Villeurbanne CEDEX, France.

2Sorbonne Université, CNRS, Institut des NanoSciences de Paris, 4 place Jussieu, 75005 Paris, France. ${ }^{3}$ Laboratoire de Réactivité de Surface, UMR CNRS 7197, Sorbonne Université, 4 place Jussieu, F-75005 Paris, France.

${ }^{4}$ Synchrotron-SOLEIL, Saint-Aubin, BP48, F91192 Gif sur Yvette Cedex, France.

${ }^{5}$ Université Paris-Saclay, CNRS, Centre de Nanosciences et de Nanotechnologies, 91120, Palaiseau, Paris, France. ${ }^{6}$ Université de Lyon, Université Claude Bernard Lyon 1, INSA Lyon, MATEIS, UMR 5510 CNRS, 7 Avenue Jean Capelle, Villeurbanne Cedex 69621, France

7 Department of Physics, University of Trento, Via Sommarive 14, 38123 Povo, Italy. 8 Graphene Labs, Fondazione Istituto Italiano di Tecnologia, Via Morego, I-16163 Genova, Italy.

*To whom correspondence should be sent: el@insp.upmc.fr and benoit.mahler@univ-lyon1.fr

\section{Table of content}

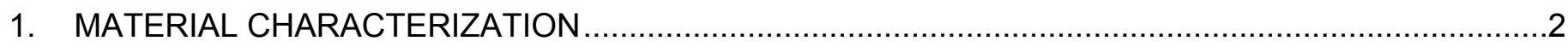

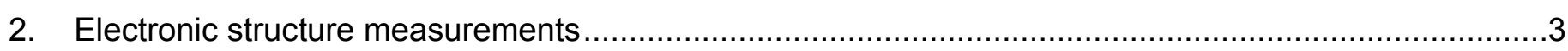

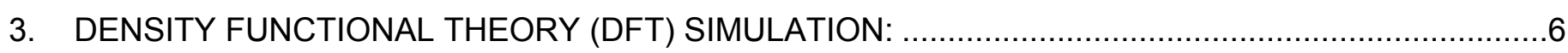

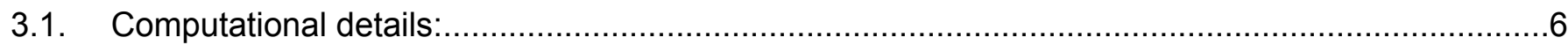

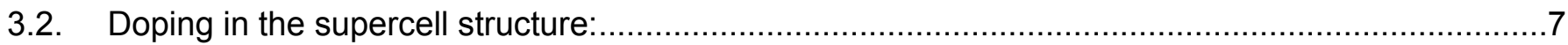

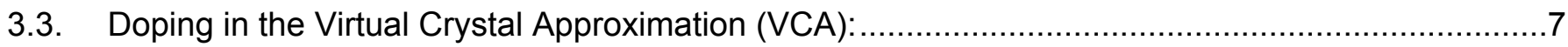

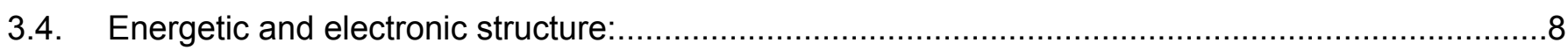

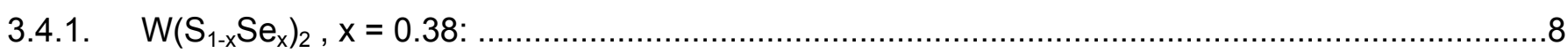

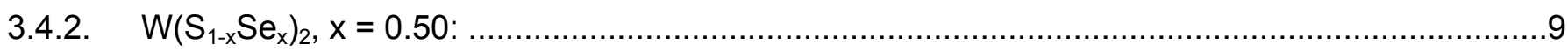

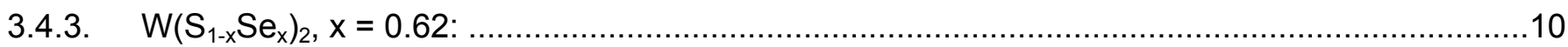

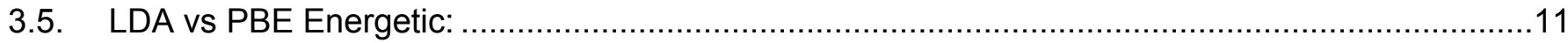

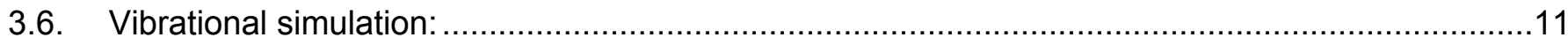

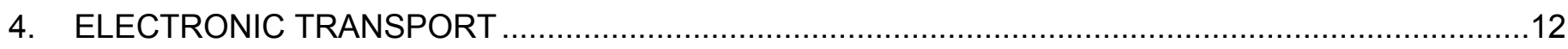

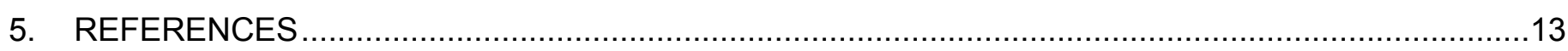




\section{MATERIAL CHARACTERIZATION}

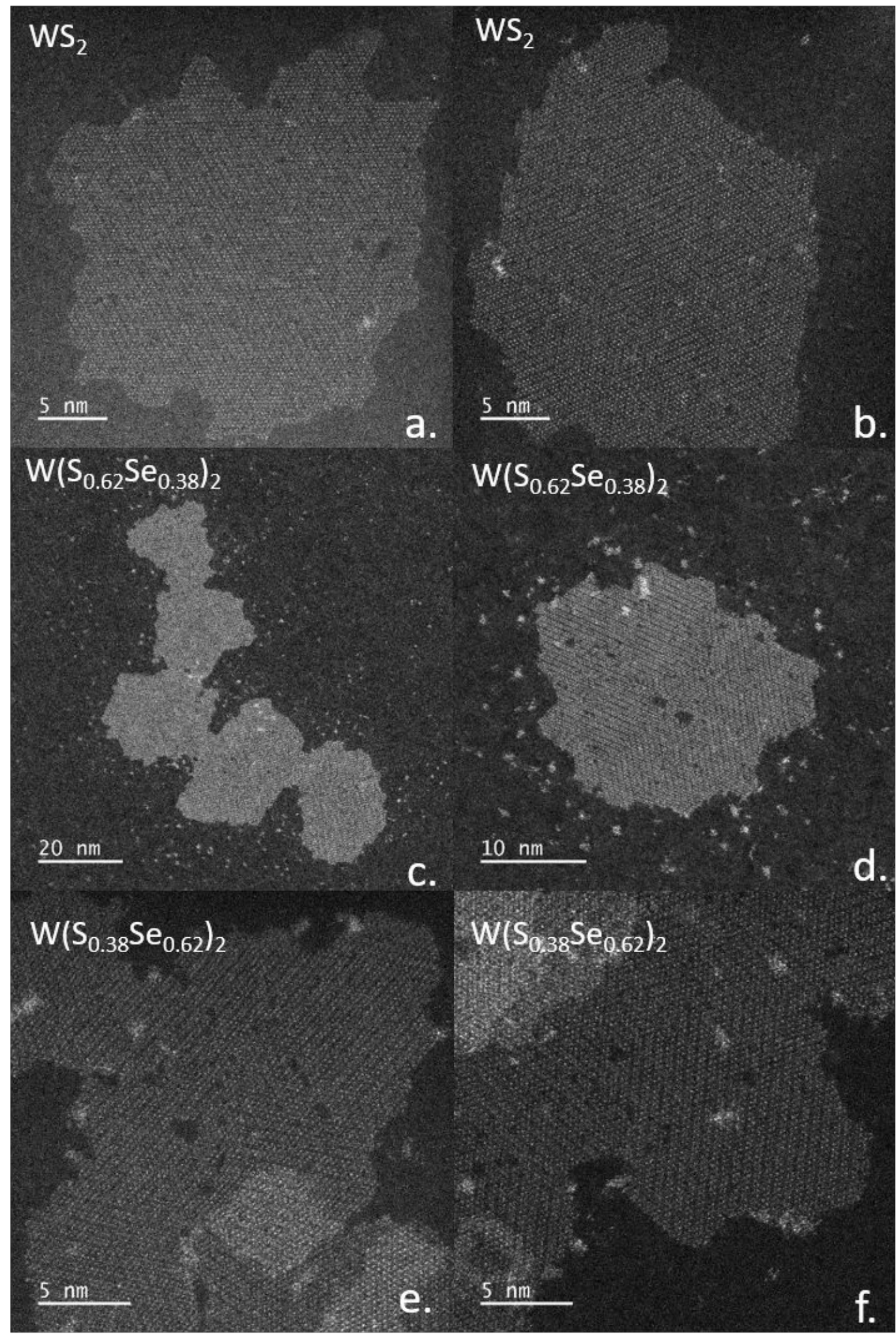

Figure S 1 high resolution transmission electron microscopy image of WSSe monolayer flakes with 1T' phase and various Se content. 


\section{Electronic structure measurements}

X-ray photoemission measurements (XPS): For photoemission, we use an epitaxial graphene substrate where graphene is obtained on the top of a SiC substrate. ${ }^{1}$ Graphene is conductive which reduce sample charging effect, while the low density of state at the Fermi level makes that no parasitic signal from the substrate is observed when acquiring the signal relative to the valence band. The sample solution was drop-casted onto this substrate and then natively dried in air. Finally, the sample was rinsed in methanol for $30 \mathrm{sec}$.

Photoemission spectra were collected on a SPECS PHOIBOS 100-1D-DLD X-ray photoelectron spectrometer, using a monochromated $\mathrm{Al} \mathrm{K}_{\alpha}(\mathrm{hv}=1486.6 \mathrm{eV})$ radiation source having a $200 \mathrm{~W}$ electron beam power. The XPS spectra are collected at pass energy of $20 \mathrm{eV}$. Valence band and secondary electron cut-off are collected at pass energy of $2 \mathrm{eV}$ using an He lamp emitting at $\mathrm{hv}=21.22 \mathrm{eV}$. Data acquisition for the cut-off is conducted while the sample is biased to $-10 \mathrm{~V}$. Photoelectrons are analyzed at a takeoff angle of $90^{\circ}$ under ultrahigh vacuum $\left(10^{-8} \mathrm{~Pa}\right)$.

Additional photoemission measurements have been conducted on the Tempo beamline of synchrotron Soleil. Samples were introduced in the preparation chamber and degassed until vacuum below $10^{-9} \mathrm{mbar}$ is reached. Then samples were introduced in the analysis chamber. The signal was acquired onto a MBS A-1 photoelectron analyzer equipped with a delay line detector developed by Elettra. ${ }^{2}$ Acquisition was done at constant pass energy (50 eV) within the detector. Photons energy of $60 \mathrm{eV}$ was used for acquisition of valence band while a $340 \mathrm{eV}$ photon energy was used for the analysis of the core level and cut off of the secondary electron. Data acquisition for the cut-off is conducted while the sample is biased under $-18 \mathrm{~V}$.
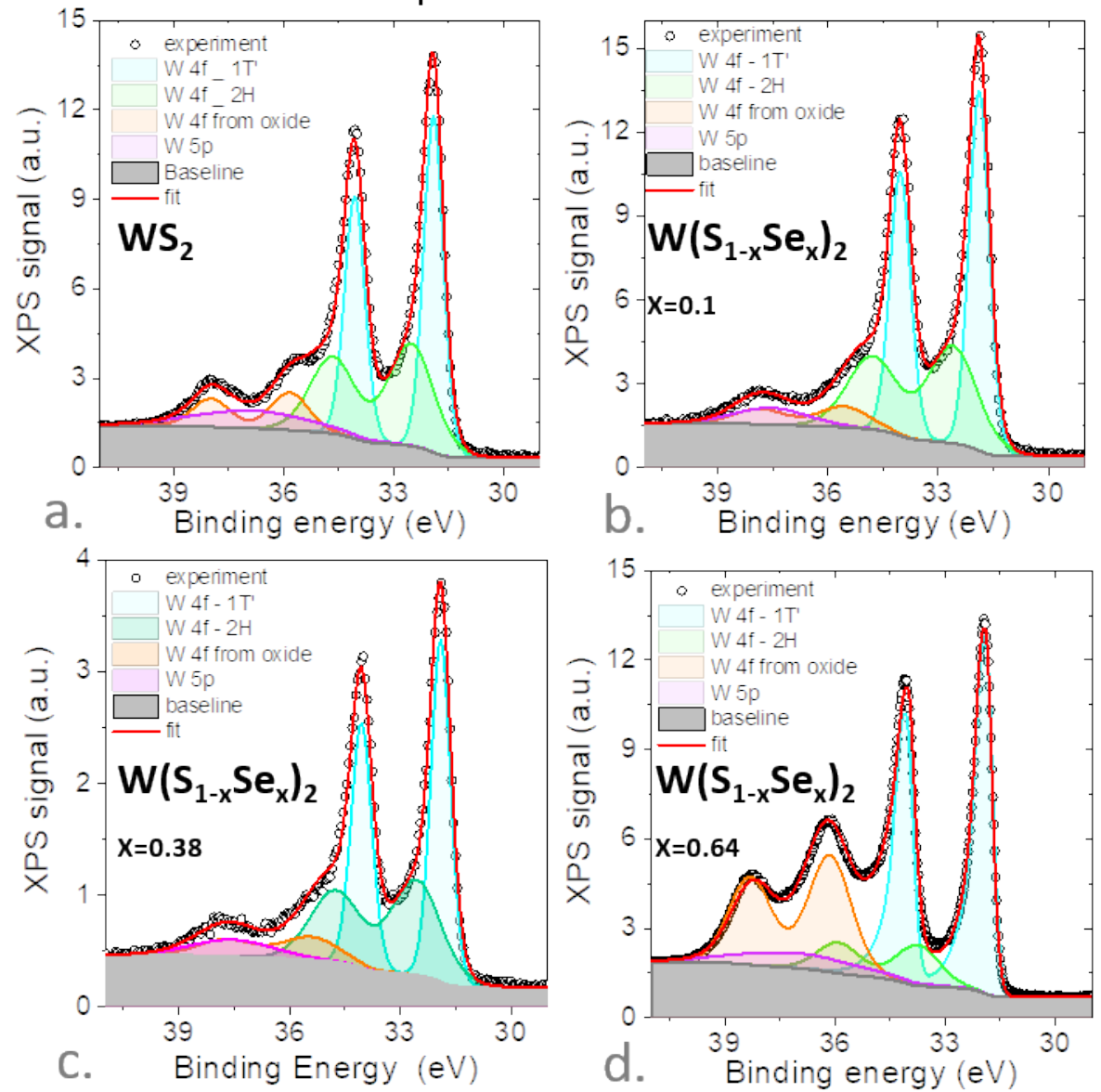

Figure S 2 Photoemission spectra relative to the W4f state for a thin film made of WSSe monolayers of $1 T^{\prime}$ phase at various Se content. 

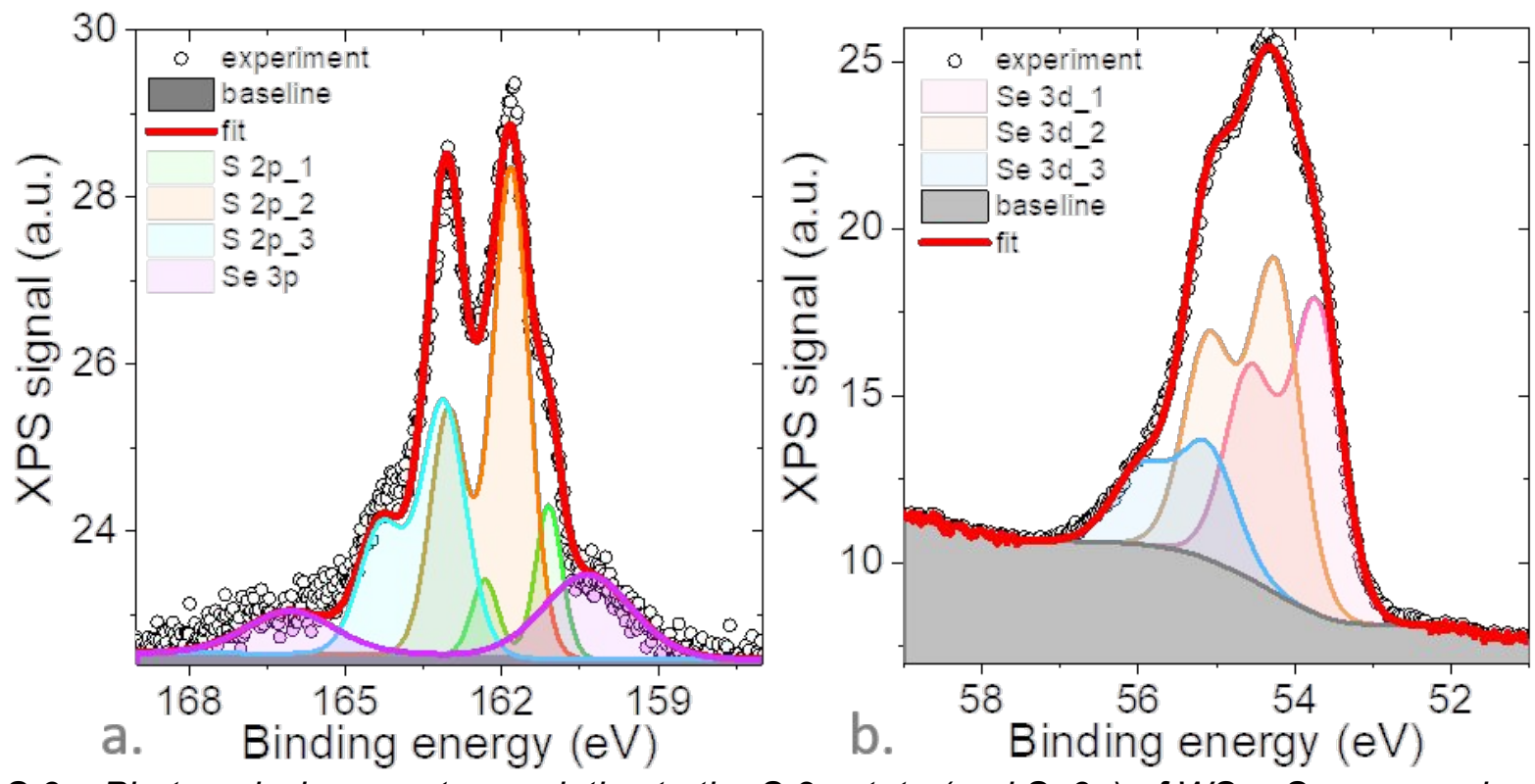

Figure $S 3 a$. Photoemission spectrum relative to the $S 2 p$ state (and $S e 3 p$ ) of $W S_{0.38} S_{0.62}$ monolayer flakes with $1 T$ 's phase. b. Photoemission spectrum relative to the Se $3 d$ state of $W S_{0.38} S_{0.62}$ monolayer flakes with $1 T$ 's phase. 

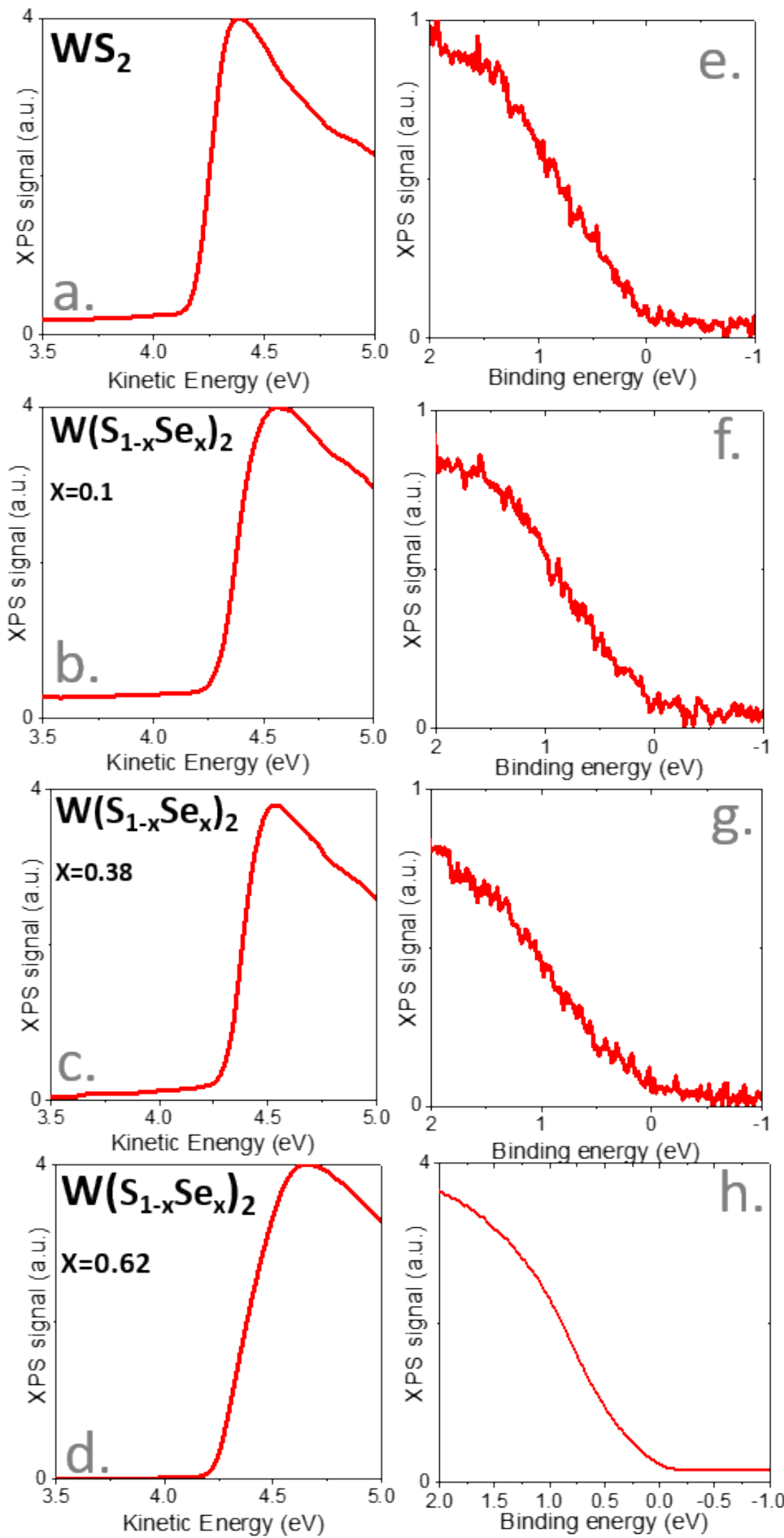

Figure $S 4$ a-d Photoemission spectrum relative to the cut-off of the secondary electrons for a thin film made of WSSe monolayers of 1T' phase at various Se content. $b$. Photoemission spectrum relative to the valence band for a thin film made of WSSe monolayers of $1 T^{\prime}$ 'phase at various Se content. 


\section{DENSITY FUNCTIONAL THEORY (DFT) SIMULATION:}

\subsection{Computational details:}

First principles calculations were performed using the plane-wave pseudopotential method as implemented in the Quantum ESPRESSO package ${ }^{3,4}$. We employed norm-conserving pseudopotentials for all atomic species, with an energy cut-off for the valence wave-function of 60 Ry. Electronic band structure and energetics for identification of the most stable doped structure were obtained through Perdew-Burke-Ernzerhof (PBE) $)^{5}$ exchange-correlation functional. Raman spectra were computed in the Local Density Approximation (LDA) by optimizing the crystal structure. The tendency of LDA to overbind leads to a somewhat better agreement with experiments although no significant differences are found in the PBE and LDA Raman active frequencies. We checked that total energy differences per W atoms were preserved when using either PBE or LDA and details can be found below.

The Brillouin-zone integration in the $2 \times 1$ supercell is performed on a uniform grid of $30 \times 60 \times 1 \mathrm{k}$ points and a first-order Methfessel-Paxton ${ }^{6}$ smearing of 0.001 Ry: these parameters assure convergence of both total energy per $\mathrm{W}$ atom and vibrational frequencies at the center of the Brillouin zone. For larger supercell, the number of k-points is then reduced according to their size. Freestanding monolayers are modelled with $\approx 12 \AA$ of vacuum between adjacent layers in the supercell and truncating the Coulomb potential along the non-periodic direction. 7

Spin-orbit coupling (SOC) effects on the electronic dispersion are also taken into account by performing non-collinear calculations with the aid of ultrasoft and fully-relativistic pseudopotentials, with cutoffs of $60 \mathrm{Ry}$ and $480 \mathrm{Ry}$ for the electronic wave-function and electronic density respectively together with a uniform grid of $30 \times 60 \times 1$ k-points and a first-order Methfessel-Paxton ${ }^{7}$ smearing of $0.001 \mathrm{Ry}$ in the $2 \times 1$ supercell. As it can be seen from Figure S 5, SOC is responsible for the topological band gap opening.

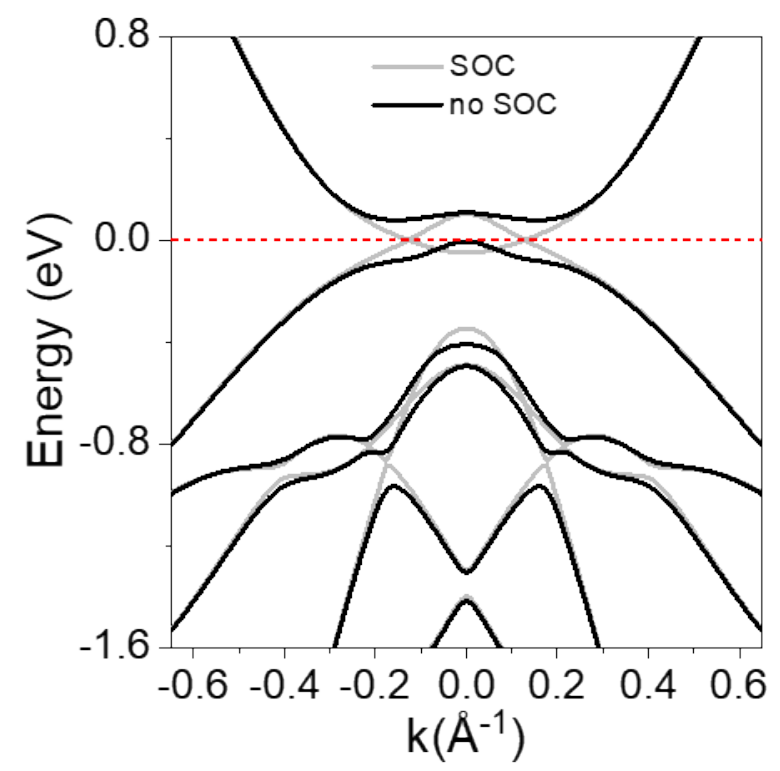

Figure $S 5$ Simulated band structure of the $1 T^{\prime}$ phase of $W S_{2}$ with and without spin orbit coupling 


\subsection{Doping in the supercell structure:}

The unit cell of $1 \mathrm{~T}^{\prime}-\mathrm{WS}_{2}$ is a $2 \times 1$ supercell containing $2 \mathrm{~W}$ and $4 \mathrm{~S}$ atoms. In order to simulate doping with variable Se content in $\mathrm{W}\left(\mathrm{S}_{1-\mathrm{x}} \mathrm{Se}_{\mathrm{x}}\right)_{2}$, we substituted $\mathrm{x} S$ atoms with $\mathrm{Se}$ ones in the $2 \times 1$, $2 \times 2,4 \times 1$ and $4 \times 2$ supercells accordingly. More specifically, for a given value of $x$ we computed the total energy of all possible non-equivalent permutations of both $S$ and Se atoms after having relaxed lattice parameters and atomic positions. In order to select the optimal structure, we chose the most favorable one by comparing the total energy per $W$ atom in the unit cell, as shown below. For each doping, the corresponding electronic dispersion relation is labelled as "ordered".

\subsection{Doping in the Virtual Crystal Approximation (VCA):}

The Virtual Crystal Approximation (VCA $)^{8}$ allows us to probe the possibility of having a disordered system upon doping by assuming that on each doped site there is a virtual atom whose chemical nature interpolates between that of the actual components. More precisely, we considered the $2 \times$ 1 supercell of $1 \mathrm{~T}^{\prime}-\mathrm{WS}_{2}$ and we substituted each $\mathrm{S}$ atom with a virtual one, whose pseudopotential has been obtained by interpolating between $(1-x) \%$ of $S$ and $x \%$ of Se pseudopotentials in order to simulate the $\mathrm{W}\left(\mathrm{S}_{1-\mathrm{x}} \mathrm{Se}_{\mathrm{x}}\right)_{2}$ system. For each doping, the corresponding electronic dispersion relation is labelled as "disordered". 


\subsection{Energetic and electronic structure:}

3.4.1. $W\left(S_{1-x} S e_{x}\right)_{2}, x=0.38$ :

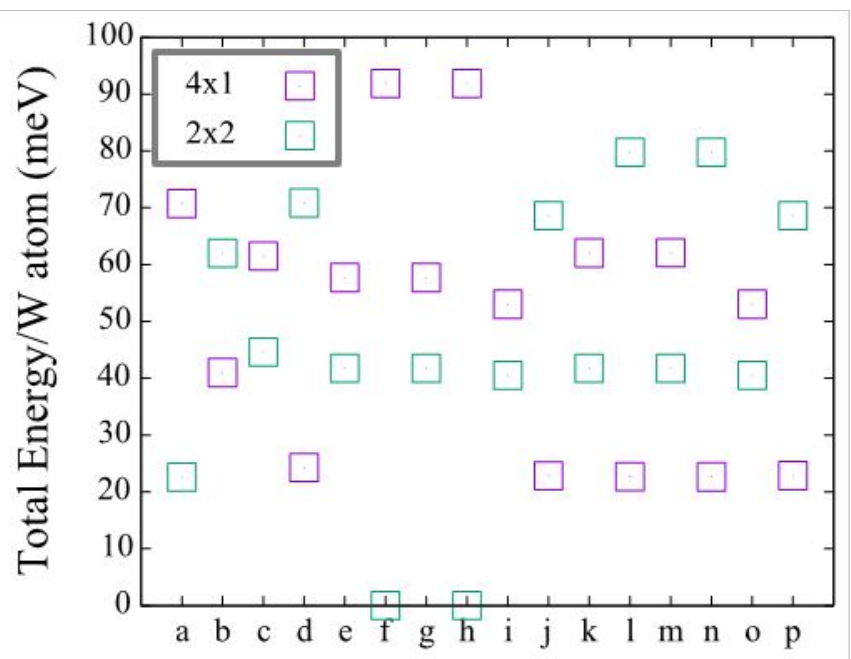

a.

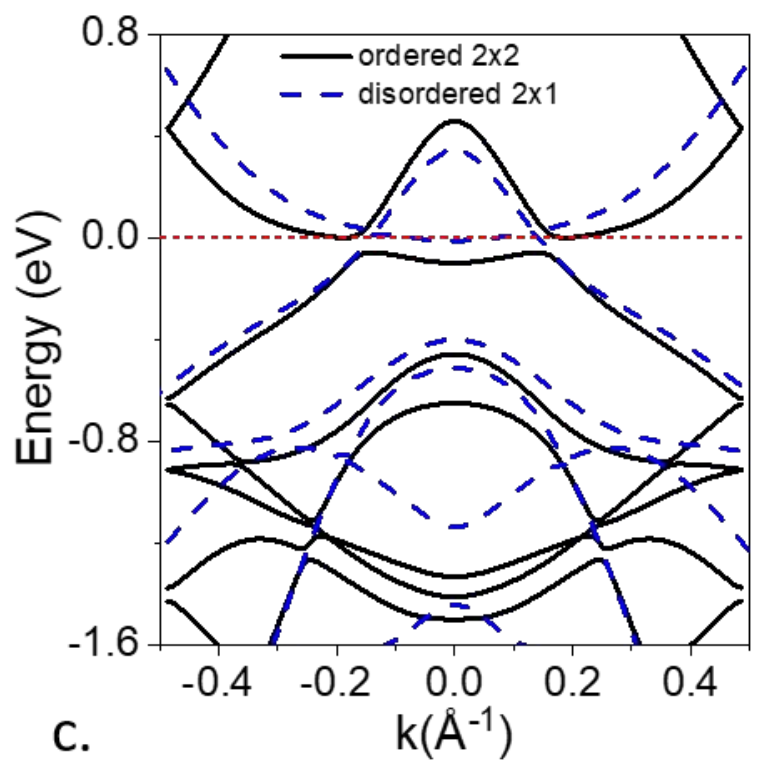

Configuration
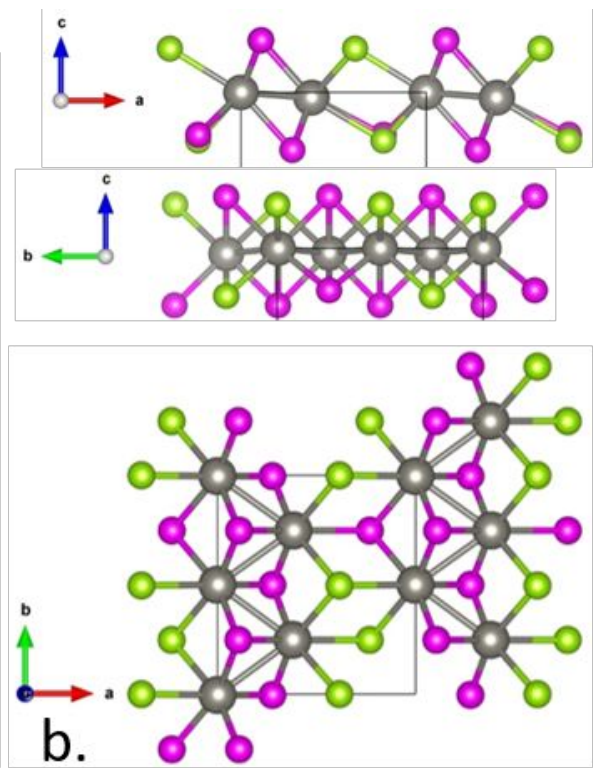

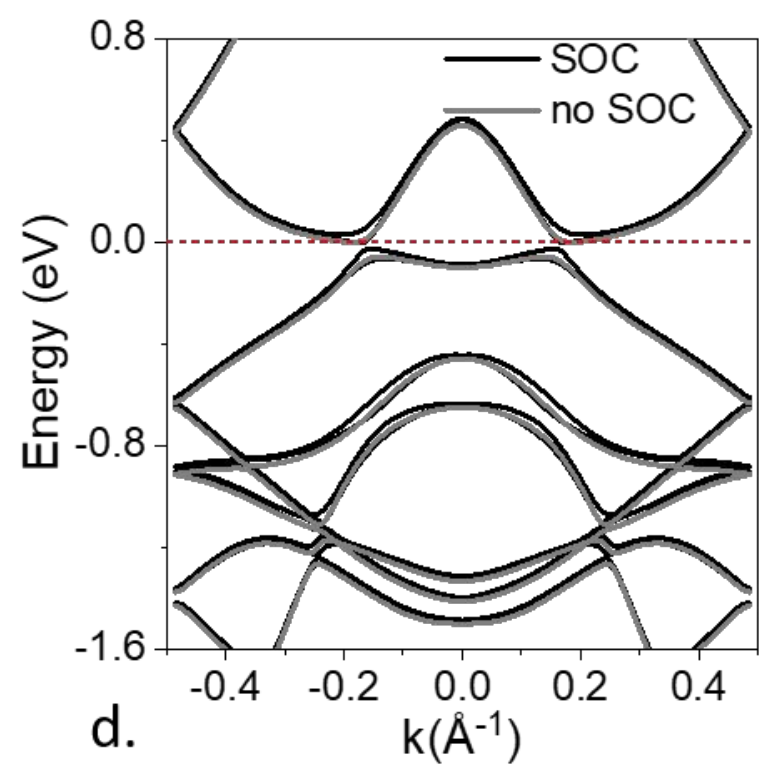

Figure S 6(a) Total energy per $W$ atom for different unit cells (4x1 and $2 \times 2)$ and for different positions of the $S$ and Se atoms. The lowest energetic configuration is set to $0 \mathrm{meV}$ and corresponds to $2 \times 2-f$. All the structures and atomic positions have been relaxed via the PBE exchange-correlation functional, as well as the energy computation; (b) Ball-and-stick model of the most energetically favored configuration. Grey, magenta and green spheres correspond respectively to W, $S$ and Se atoms; (c) Electronic dispersion relation of $W S_{0.62} S_{e_{0.38}}$. Black solid line corresponds to the ordered $2 \times 2-f$ configuration, i.e. the most stable one obtained introducing Se atoms according to the doping. Blue dashed line corresponds to the disordered $2 \times 1$ configuration, i.e. doping is simulated in virtual crystal by introducing fictitious atoms in the $2 \times 1$ unit cell. Electronic band structure has also been computed via the PBE exchange-correlation functional. (d) Simulated band structure of the $1 T^{\prime}$ phase of $W S_{0.38} S_{e_{0.62}}$ for the ordered structure with and without spin orbit coupling. 
3.4.2. $W\left(S_{1-x} S e_{x}\right)_{2}, x=0.50$ :
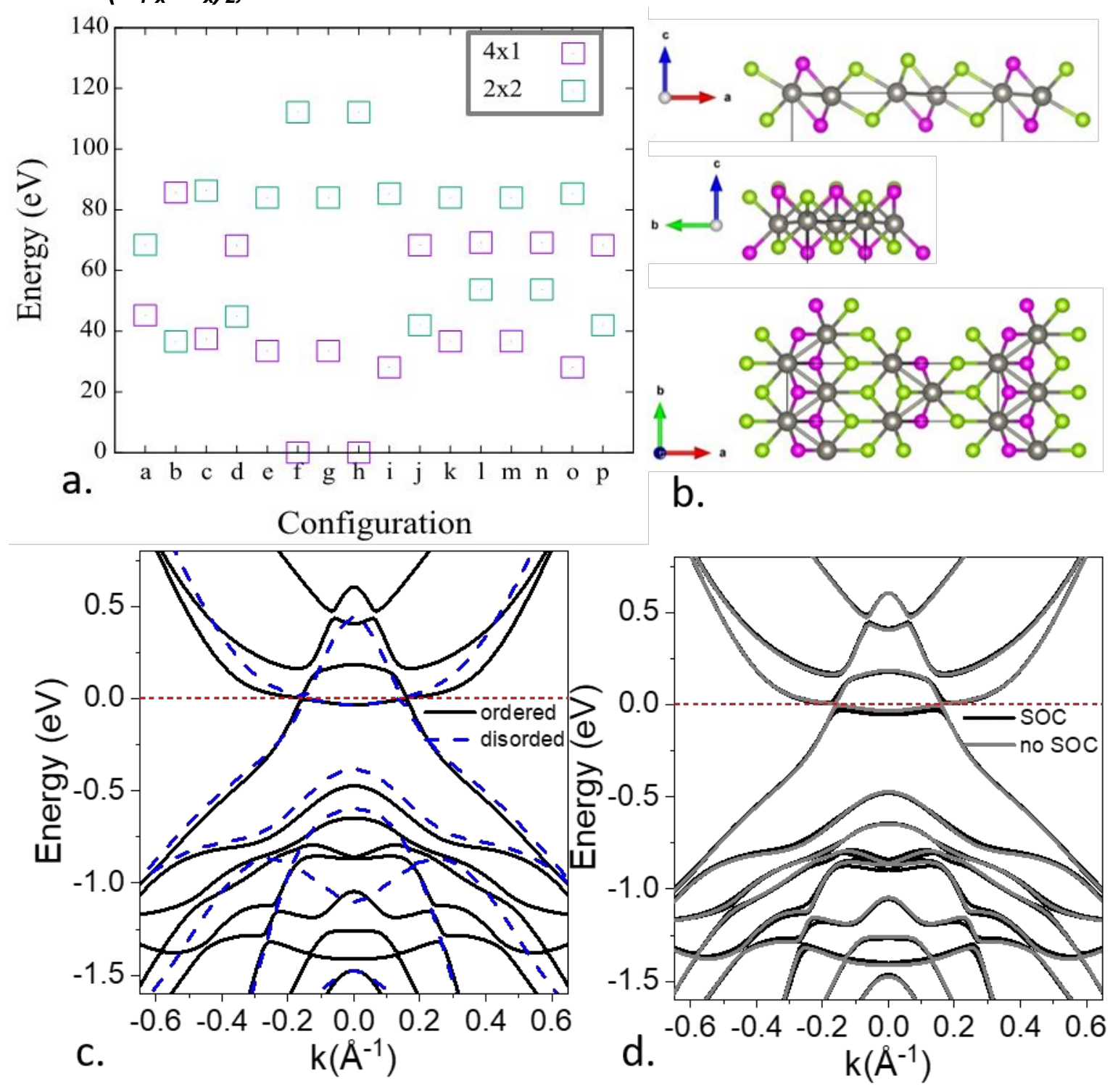

Figure $S 7($ a) Total energy per $W$ atom for different unit cells $(2 \times 1,4 \times 1,2 \times 2$ and $4 \times 2)$ and for different positions of the $S$ and Se atoms. The lowest energetic configuration is set to $0 \mathrm{meV}$ and corresponds to $2 \times 1-c$. All the structures and atomic positions have been relaxed via the PBE exchange-correlation functional, as well as the energy computation; (b) Ball-and-stick model of the most energetically favored configuration. Grey, magenta and green spheres correspond respectively to W, $S$ and Se atoms; (c) Electronic dispersion relation of $W S_{0.50} S_{e_{0.50}}$. Black solid line corresponds to the ordered $2 \times 1-c$ configuration, i.e. the most stable one obtained introducing Se atoms according to the doping. Blue dashed line corresponds to the disordered $2 \times 1$ configuration, i.e. doping is simulated in virtual crystal by introducing fictitious atoms in the $2 \times 1$ unit cell. Electronic band structure has also been computed via the PBE exchange-correlation functional. (d) Simulated band structure of the $1 T^{\prime}$ phase of $W S_{0.5} S_{0.5}$ for the ordered structure with and without spin orbit coupling 
3.4.3. $W\left(S_{1-x} S e_{x}\right)_{2}, x=0.62$ :
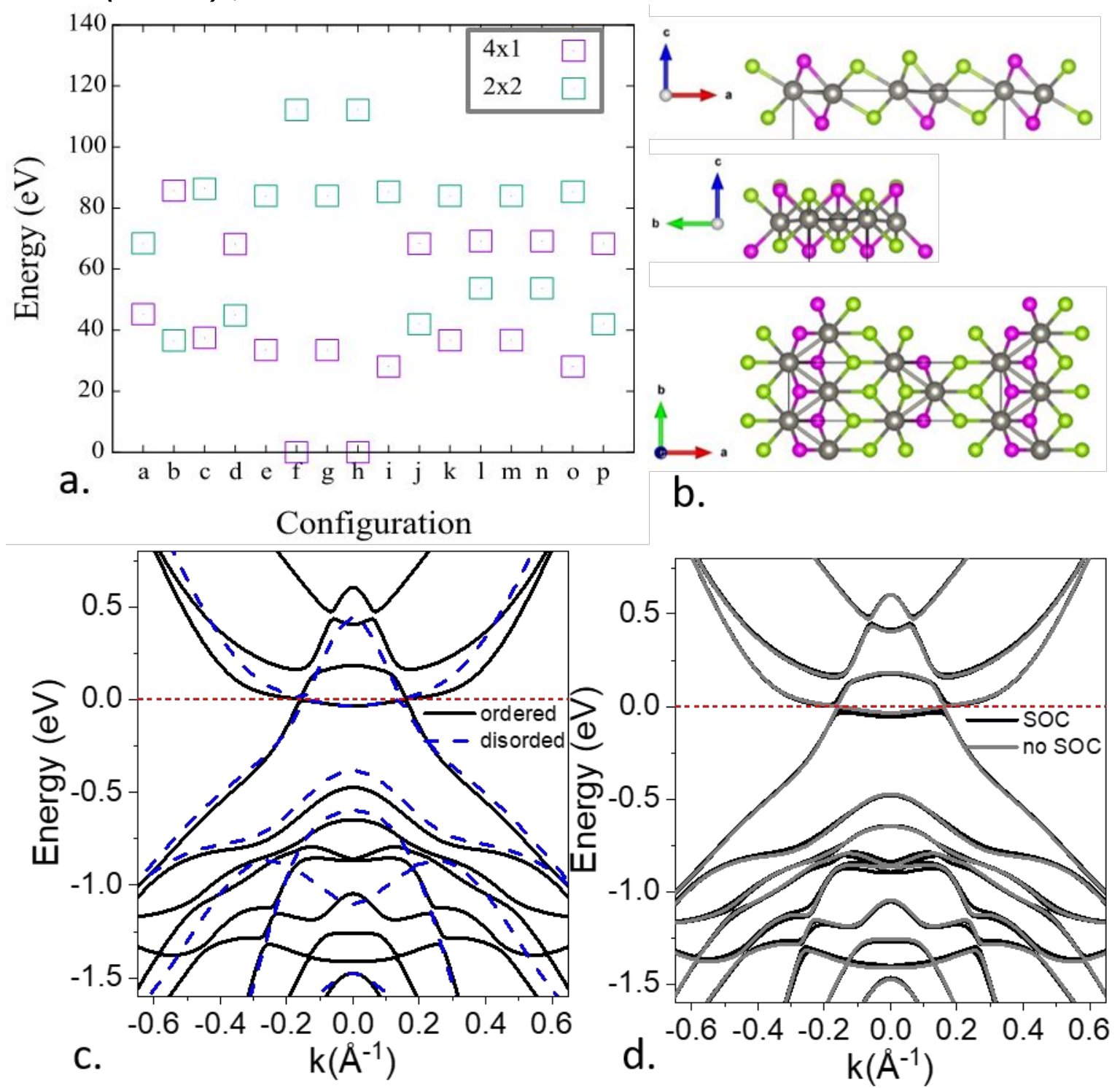

Figure $S$ 8(a) Total energy per $W$ atom for different unit cells (4x1 and $2 x 2)$ and for different positions of the $S$ and Se atoms. The lowest energetic configuration is set to $0 \mathrm{meV}$ and corresponds to $4 x 1-f$. All the structures and atomic positions have been relaxed via the PBE exchange-correlation functional, as well as the energy computation; (b) Ball-and-stick model of the most energetically favored configuration. Grey, magenta and green spheres correspond respectively to W, $S$ and Se atoms; (c) Electronic dispersion relation of $W S_{0.38} S_{e_{0.62}}$. Black solid line corresponds to the ordered $4 \times 1-f$ configuration, i.e. the most stable one obtained introducing Se atoms according to the doping. Blue dashed line corresponds to the disordered $2 \times 1$ configuration, i.e. doping is simulated in virtual crystal by introducing fictitious atoms in the $2 \times 1$ unit cell. Electronic band structure has also been computed via the PBE exchange-correlation functional. (d) Simulated band structure of the $1 T^{\prime}$ phase of $W S_{0.38} S_{e_{0.62}}$ for the ordered structure with and without spin orbit coupling 


\subsection{LDA vs PBE Energetic:}

Table $S 1$ Energy difference between the highest and the lowest ordered configuration as a function of doping $x$ for both the LDA and PBE exchange-correlation functional. More precisely, given a doping $x$, the energy difference is defined as: $\Delta E_{x}=E_{x}^{(H)}-E_{x}^{(L)}$, where $E_{x}^{(H)}$ and $E_{x}^{(L)}$ are respectively the highest and lowest total energy per $W$ atom for doping $x$.

\begin{tabular}{llll}
\hline \hline FUNCTIONAL & $\Delta \mathrm{E}_{0.38}(\mathrm{meV})$ & $\Delta \mathrm{E}_{0.50}(\mathrm{meV})$ & $\Delta \mathrm{E}_{0.62}(\mathrm{meV})$ \\
\hline LDA & 93.12 & 97.39 & 112.26 \\
\hline PBE & 91.86 & 95.57 & 112.13 \\
\hline \hline
\end{tabular}

\subsection{Vibrational simulation:}

The LDA exchange-correlation potential has been chosen in order to best reproduce experimental results for pristine $1 \mathrm{~T}^{\prime}-\mathrm{WS}_{2}$. The height of the peaks does not correspond to the actual Raman intensities, they are just placeholders for identifying the Raman active modes.

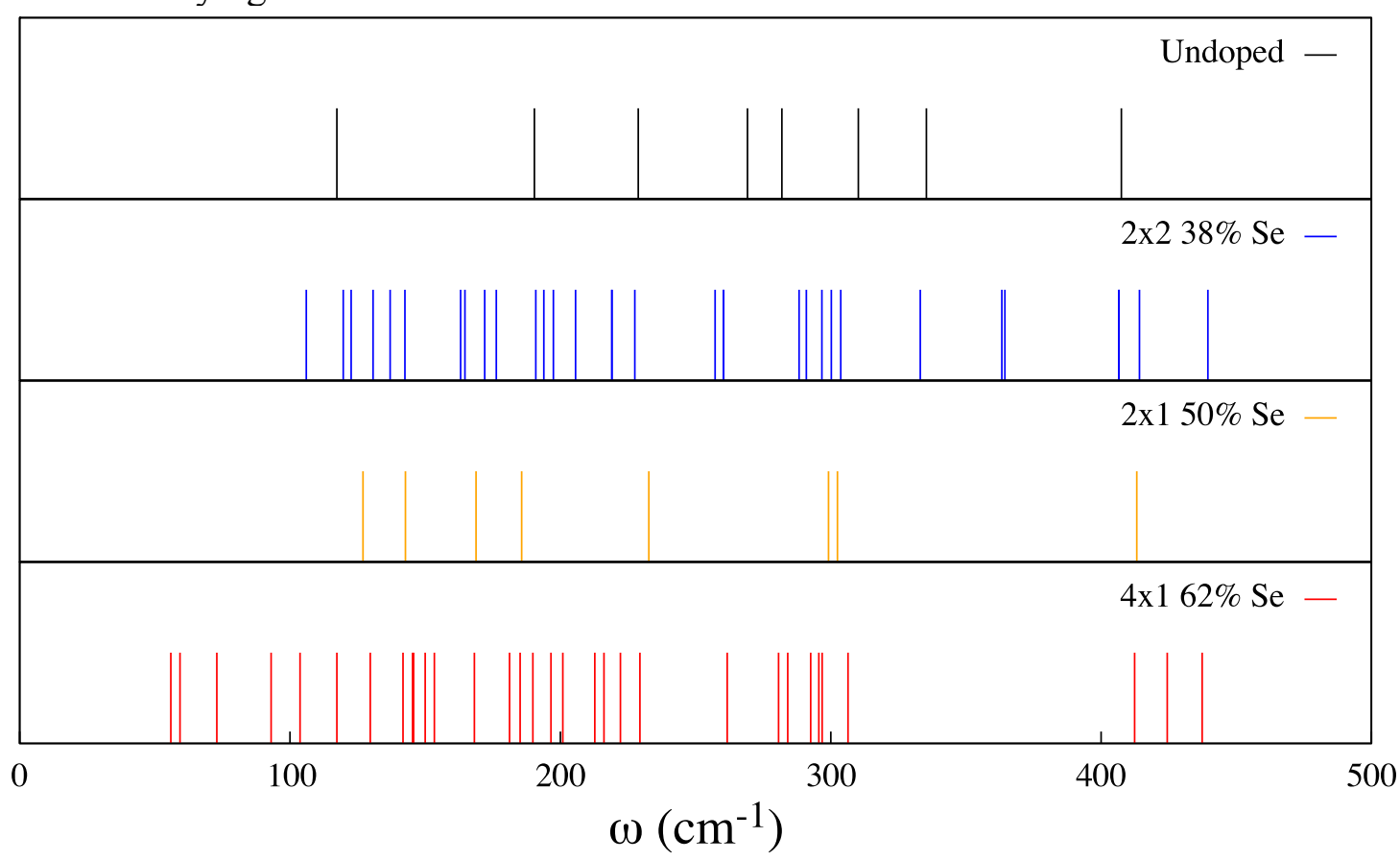

Figure $S 9$ Ab-initio Raman spectra computed via the $L D A$ exchange-correlation functional for $W\left(S_{1-x} S e_{x}\right)_{2}$, after having relaxed the crystal structure. Undoped correspond to $x=0$, while $38 \%, 50 \%$ and $62 \%$ Se correspond to $x=0.38, x=0.50$ and $x=62$ respectively. Raman spectra are computed for the ordered structures. 


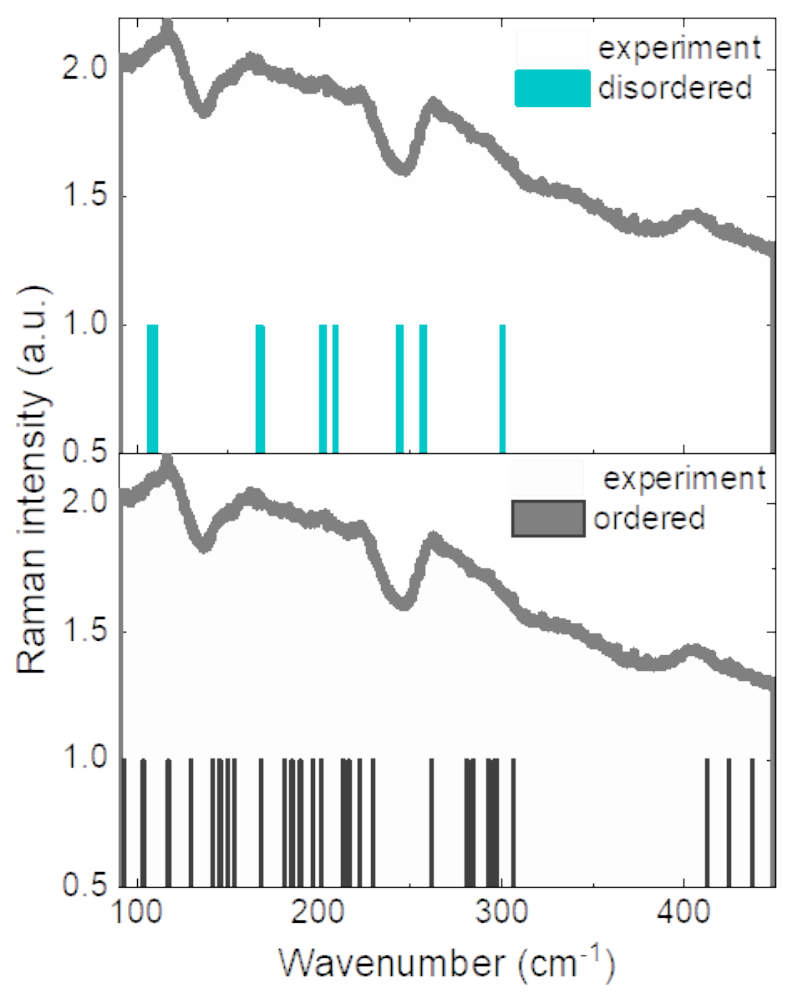

Figure $S 10$ Experimental (thick grey line) and simulated (grey histogram for the ordered phase and cyan for the disordered phase) Raman spectra for $1 T^{\prime}$ phase of $W S_{0.38} S_{0.62}$.

\section{ELECTRONIC TRANSPORT}

In the main text we have plotted th temperature depedence of the current as a function of $\mathrm{T}^{-0.5}$, which correspond to Efros-Shklovskii's model. here we provide other scaling, such as $\mathrm{T}^{-0.25}$ (Mott's model) and $\mathrm{T}^{-0.33}$ (expected scaling in a purely $2 \mathrm{D}$ hopping)
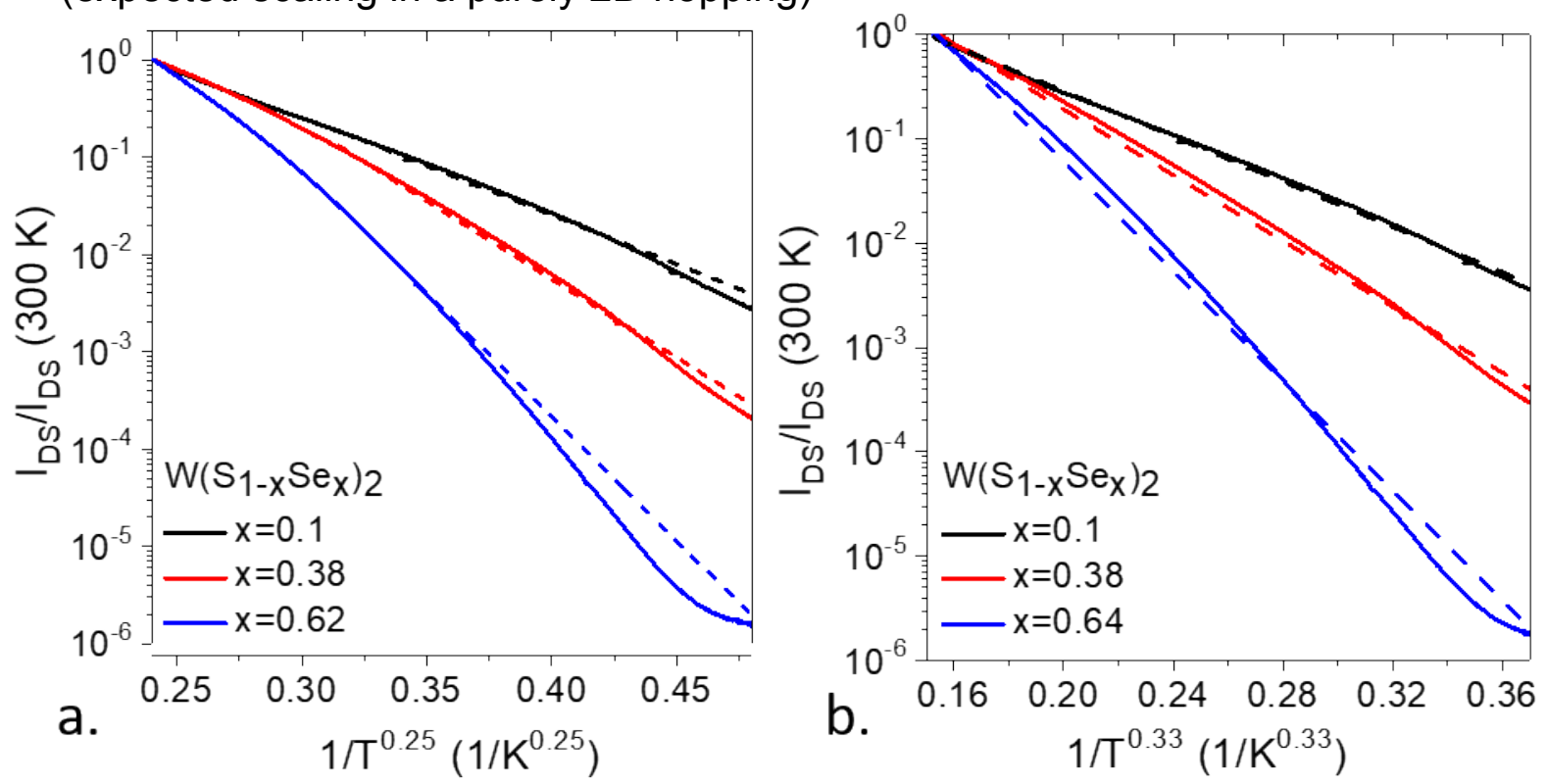

Figure S 11 a. Current as a function of temperature for WSSe monolayer flakes with 1T's phase with various Se content. The dashed lines are obtained using Mott's fit with the following expression$\sigma \propto \exp \left(-\left(T_{M} / T\right)^{1 / 4}\right)$. b. Same set of data where the temperature is this time plotted with $T^{-0.33}$ scaling. Dashed lines are single exponential fit. 


\section{REFERENCES}

(1) Pallecchi, E.; Lafont, F.; Cavaliere, V.; Schopfer, F.; Mailly, D.; Poirier, W.; Ouerghi, A. High Electron Mobility in Epitaxial Graphene on $4 \mathrm{H}-\mathrm{SiC}(0001)$ via Post-Growth Annealing under Hydrogen. Sci. Rep. 2014, 4, 4558.

(2) Bergeard, N.; Silly, M. G.; Krizmancic, D.; Chauvet, C.; Guzzo, M.; Ricaud, J. P.; Izquierdo, M.; Stebel, L.; Pittana, P.; Sergo, R.; et al. Time-Resolved Photoelectron Spectroscopy Using Synchrotron Radiation Time Structure. J. Synchrotron Radiat. 2011, 18, 245-250.

(3) Giannozzi, P.; Andreussi, O.; Brumme, T.; Bunau, O.; Nardelli, M. B.; Calandra, M.; Car, R.; Cavazzoni, C.; Ceresoli, D.; Cococcioni, M.; et al. Advanced Capabilities for Materials Modelling with Quantum ESPRESSO. J. Phys. Condens. Matter 2017, 29, 465901.

(4) Giannozzi, P.; Baroni, S.; Bonini, N.; Calandra, M.; Car, R.; Cavazzoni, C.; Ceresoli, D.; Chiarotti, G. L.; Cococcioni, M.; Dabo, I.; et al. QUANTUM ESPRESSO: A Modular and OpenSource Software Project for Quantum Simulations of Materials. J. Phys. Condens. Matter 2009, 21, 395502.

(5) Perdew, J. P.; Burke, K.; Ernzerhof, M. Generalized Gradient Approximation Made Simple. Phys. Rev. Lett. 1996, 77, 3865-3868.

(6) Methfessel, M.; Paxton, A. T. High-Precision Sampling for Brillouin-Zone Integration in Metals. Phys. Rev. B 1989, 40, 3616-3621.

(7) Sohier, T.; Calandra, M.; Mauri, F. Density Functional Perturbation Theory for Gated TwoDimensional Heterostructures: Theoretical Developments and Application to Flexural Phonons in Graphene. Phys. Rev. B 2017, 96, 075448.

(8) Bellaiche, L.; Vanderbilt, D. Virtual Crystal Approximation Revisited: Application to Dielectric and Piezoelectric Properties of Perovskites. Phys. Rev. B 2000, 61, 7877-7882. 